\title{
Characterising Computer Experience and Anxieties Differences Between Middle Eastern and Western Students in eLearning
}

\author{
Raafat George Saadé, Fassil Nebebe, and Dennis Kira \\ John Molson School of Business, \\ Concordia University, Montreal, Canada
}

\section{raafat.saade@concordia.ca; fassil.nebebe@concordia.ca; dennis.kira@concordia.ca}

\begin{abstract}
Computer anxiety especially in first-year university students is common and continues to be a significant issue as it manifests itself in a number of negative emotions with significant detrimental impact to students. This is especially true in the context where students use computers for course work - online learning. Research work addressing the effect of computer/Internet experience on anxiety as it differs across cultures is relatively scarce. In this study, we investigate online learning anxiety and how it is impacted by computer and the internet experience, across two cultures - Middle Eastern and Westen. A survey methodology approach was used for the capture of data and analysis. We found that Anxiety using computers for learning still exists in today's time across both cultures, however the type of anxiety differs between them. At the same time computer/Internet experience was found to impact anxiety in English student group while amount of computer usage was found to influence anxiety in the ME group. Conclusions, limitations and recommendations for future research are provided.
\end{abstract}

Keywords: Anxiety, Computer Use, Computer Experience, Culture, Middle East, Online Learning.

\section{Introduction}

Modern communications outlined by the Internet has changed all our facets of our lives and transformed our world into ubiquitous connectivity: Anytime and anywhere. Online learning rate continues to increase, and more researchers, practitioners and institutions have begun to realize its value. However, online learning remains a challenge even though that many models have been

Material published as part of this publication, either on-line or in print, is copyrighted by the Informing Science Institute. Permission to make digital or paper copy of part or all of these works for personal or classroom use is granted without fee provided that the copies are not made or distributed for profit or commercial advantage AND that copies 1) bear this notice in full and 2) give the full citation on the first page. It is permissible to abstract these works so long as credit is given. To copy in all other cases or to republish or to post on a server or to redistribute to lists requires specific permission and payment of a fee. Contact Publisher@,InformingScience.org to request redistribution permission. used to measure technology usage, effectiveness and satisfaction yet relatively few are in the online learning context (Aldalalah \& Gasaymeh, 2014).

Internet information technologies have been used to play an important role in the learning process. To that effect, a lot of effort has been devoted to creating online learning environments using different management systems: document 
management systems, content management systems, and learning management systems. In the past decade, a number of studies have pointed out that factors associated with online learning; however the focus of most of these studies were either on satisfaction in using a specific learning management system, or comparative (as student experience and perceptions may differ from face to face classes) and descriptive in nature.

Taking the cue from other researchers and contributing to the scarce research in cultural differences in online learning, we focus in this study on anxiety (ANX) as it differs between Middle Eastern and Western cultures, both groups taking the same online course in Canada. In the following section we review the theoretical background of anxiety as it relates to differnet contexts such as computer experience.

Following Saadé and Kira (2009), and considering the various reported definitions of anxiety, as well as the different contexts we find that anxiety is treated as a set of the following emotions: fearfulness, apprehension, and uneasiness. In our research, we view anxiety in online learning as "a feeling of fear form misuse of information technology compromising course performance". In that sense, it is not about uneasiness and apprehension, but simply about making error with information technology where the cost of the error is perceived to be high - lower grade.

\section{Literature Review}

Prior research which has been empirically supported indicates that computer and internet experience leads to the formation of positive attitudes toward computer (Al-Gahtani, 2014, Saadé \& Kira, 2007). In addition, it has been reported that student experiences are expected to have negative impact on anxiety levels in general and computer anxiety in specific and help temper student's anxiety levels in using computers for course work (McInerney et al. 1994). There seems to be a viable relationship between measures of computer experience, computer anxiety and confidence as reported by much research since the time that the availability of computers became widespread in $90 \mathrm{~s}$. Ever since, measuring of computer experience has been primarily done subjectively by asking the amount of computer use; how many years the students have used a computer; and for how many hours a week do they use a computer and for which purpose.

Computer experience has been viewed as an individual difference factor that influences a student's formation of beliefs about using a system for the purpose of learning. The extent of experience gained by using directly computers in a variety of ways and purposes affects the student's perception of the system's utility for learning, thereby influencing their attitude and anxiety levels, which in turn provides positive support towards their performance. As it relates to learning experience and performance, anxiety is one of the most important sources of information about the system's effectiveness. To that effect, more research in this area needs to be considered to better understand the effects of computer experience as an individual difference, on anxiety.

Past computer usage studies revealed computer usage experience as a common thread linking computers to the students. Generally speaking, these findings tend to be supportive of intuitively appearing proposition that a positive attitude toward computers is associated with greater computer experience. This is a misconception as other work has shown that the type of computer experiences is equally important in forming student's attitudes and anxiety levels. A detailed account of findings in this line of research can be found in Saadé \& Kira (2007) and we only summarize some of the most important ones here in point form:

- In end-user computing research: students with more computer experience had higher levels of computer skill,

- Computer experience has been shown to have a positive effect on computer attitudes,

- Computer usage was found to significantly differ between experienced and inexperienced users 
- For experienced users, there was a stronger link between intentions and usage,

- As direct experience with technology increases over time, individuals have a better assessment of the benefits and costs associated with using that technology

- Computer experience will directly and indirectly affect usage through beliefs and that individual skills,

- Computer usage expertise was related to user beliefs and usage.

- The use of computer technology depends on the technology itself and the level of skill or expertise of the individual using it,

- The relationship between experience, expressed as skills or expertise, was empirically,

- Past computer usage influences the ease of use of the system, and is the key factor in determining future usage.

Technology usage in general often has unpleasant side effects such as strong negative emotional states as a result of the interaction process. Frustration, confusion, anger, anxiety and similar emotional states are just to name a few, and can affect not only the interaction itself, but also productivity, learning, social relationships, and overall well-being. The literature provides a number of definitions explaining anxiety, but we will not elaborate herein because it is out of the scope of the present research work. Factors to determine anxiety such as first time introduction to computer technology (Rosen et al, 1987; Brosnan, 1998), past failure/successes manipulating hardware or software; and the current tasks being attempted including the new computer applications, have been studied. In those studies researchers have attempted to predict those who will experience computer anxiety and such factors as age, gender, ethnicity, previous computer experience, mathematics anxiety, self-efficacy, learning styles, and computer attitude are posited as a factor influencing computer anxiety (Reed and Ayersman, 1996; Ayersman \& Reed, 1995; Igbaria \& Chakrabarti, 1990).

Based on prior research on computer experience and its influence on overall student's attitudes, perceptions and anxiety levels, and the fact that there exists a gap in the body of knowledge on how this differs between cultures, we attempt herein to increase our understanding of computer usage and anxiety between two distinct groups of students identified as the Middle Eastern Group and the Western Group.

\section{Methodology}

A survey methodology approach was used. The context and process including participants and procedure and the questionnaire are elaborated.

\section{Context of Study}

The study was conducted in an undergraduate course (management of information systems, MIS) at a major university in Canada, spanning three semesters (one year), using a learning tool (developed in-house) as the target system. This learning tool is a web-based information system that is accessed and used via any browser on any platform (PC or Mac).

Throughout each semester, students in this introductory course used the learning tool as part of the course learning and assessment instructional strategy. The objective of the learning tool was to test their understanding of the course subject matter. This was done by practicing multiple choice and true or false questions. The subject matter of the course entailed the following: Hardware, software, networks and the Internet, enterprise information systems, ecommerce and ebusiness, ethics in MIS, database management, acquiring information systems, information technology governance and information systems security and privacy management. The learning goals of the course were: 
- To familiarize students with an appreciation for the technical jargon of IS/IT in order to facilitate communication between you and IS/IT professionals,

- To expose students to various issues surrounding the management of IS/IT and to help them understand the strategic implications of IS/IT,

- Demostrate how information systems can enhance business decision making,

- To provide students with practical knowledge of some basic MIS tools such as the entity relationship diagram, the data flow diagram, and Microsoft Excel and Access.

The web-based learning tool monitored the students' activities by storing their login and logout times (time spent using the system) the chapters practiced, and scores. The learning tool was programmed to be as simple as possible to use and was made up of the student user interface, the student interactive session manager which stored and controled (logic) the interaction data and the knowledge database which contained all the questions and results. The front end is simple and allows the student to log into the web site and select whether he/she wants to practice or get evaluated. The interaction session manager keeps track of the student's performance as well as controls the logic behind the selection of the questions from the database and the manner of prompting them to the student. The database contains the multiple choice and true or false pools of questions, students' answers to the questions and time that they spent answering each set of questions.

The learning tool included a limited number of questions for each chapter (subject matter) (eg. chapter 1 had 38, while chapter 2 112). The student experience using the tool is:

1. Login.

2. Select practice or test.

3. Select chapter / subject.

4. System promtps student with a set of five questions at a time.

5. Student answers the five questions, and click on 'evaluate'. The system will show the correct/wrong answer with a green/red button on the side of each question.

6. Student clicks on 'next' button and system randomizes and presents another set of 5 questions.

7. Student can go back to change the subject matter, and practice accordingly.

8. When student is ready, he/she can go back to main menu and select test followed by subject/chapter and do the test.

This learning tool pedagogy (epedagogy) allows the questions to re-appear to the student. The repetition of the questions, combined with immediate feedback re-inforces student's short-term memory, recognition, and recollection abilities. When a question is repeated, a student will either get the answer right or wrong. A correct second attempt to answer a question reinforces the students' understanding of the question and of the concept at hand. An incorrect second attempt to answer a question 'jolts' student's attention and reinforces the students' reasoning abilities of the knowledge embedded in the question-answers set and of the concept at hand. The process is therefore 'cognitivist' and works at different levels.

As part of the instructional strategy, students are asked to do a minimum of 20 questions to meet the course requirements, but encouraged to do as many as they feel necessary. Students are asked to develop their own strategies of use of this tool, thereby promoting self-directed learning and driving the message that students are also responsible for their learning process. In other words, they are given tools to learn and guidance, but they must develop the strategy that suits them best - in essence providing students with the opportunity of self reflection. Students are allowed to practice in groups and by referring to any resources and materials. The objective is more to have them engaged in the processing of domain content rather than assessment. 


\section{Survey \& Procedures}

At the end of each semester, a survey instrument (table 1) was administered. Over the span of three semesters a total of 648 students participated in the study. Considering the total 648 participants, the respondents were very close to 50:50 between male and female with a mean age of 23 years, and an average of 2 years of work experience. The participants reported to use the Internet to have a strong knowledge of basic software utilization. In this study, out of the 648 participants, 54 were from Middle Eastern (ME) origin and 217 were Western (W). The grouping was done by asking the mother tongue and ME group were selected from those who answered Arabic while W from English. In the following analysis, to remain true to the data, we refer to the groups using their mother tongue answers.

Table1. Items and constructs used in the study.

\begin{tabular}{|c|c|c|}
\hline Construct & Item & Measure \\
\hline \multirow[t]{4}{*}{ Anxiety (ANX) } & ANX1 & I feel apprehensive about using computers. \\
\hline & ANX2 & $\begin{array}{l}\text { It scares me to think that I could cause the computer to destroy a } \\
\text { large amount of information by hitting the wrong key. }\end{array}$ \\
\hline & ANX3 & $\begin{array}{l}\text { I hesitate to use a computer for fear of making mistakes I cannot } \\
\text { correct. }\end{array}$ \\
\hline & ANX4 & Computers are somewhat intimidating to me. \\
\hline $\begin{array}{l}\text { Computer Knov } \\
\text { How would you } 1\end{array}$ & Computer Knowledge (CK) & ith respect to your knowledge about computers? \\
\hline \multicolumn{3}{|c|}{$\begin{array}{l}\text { OVery Knowledgeable } \\
\text { OSomewhat knowledgeable } \\
\text { ONeither knowledgeable nor unknowledgeable } \\
\text { OSomewhat unknowledgeable } \\
\text { OVery unknowledgeable }\end{array}$} \\
\hline \multicolumn{2}{|c|}{ How long have you been using the internet? } & the internet? \\
\hline $\begin{array}{l}\text { OLess than } 6 \text { mo } \\
\text { OBetween } 6 \text { mor } \\
\text { O1+ to } 2 \text { years } \\
\text { O2+ to } 5 \text { years } \\
\text { O More than } 5 \text { y }\end{array}$ & and 1 ye & \\
\hline \multicolumn{3}{|c|}{ Time Daily of Internet Usage (TDIUse) } \\
\hline $\begin{array}{l}\text { OLess than } 15 \mathrm{~m} \\
\text { OBetween } 15 \mathrm{mi} \\
\text { O1+ to } 2 \text { hours } \\
\text { O2+ to } 5 \text { hours } \\
\text { OMore than } 5 \text { ho }\end{array}$ & $\begin{array}{l}\text { es } \\
\text { s and } 11\end{array}$ & \\
\hline
\end{tabular}


Items (presented in Table 1) used to measure the constructs of anxiety (ANX), computer experience (CE) and internet experience (IE) were adopted from prior research work namely Davis (1989) and Venkatesh (1999). The adapted the validated items where some wording was changed to account for the context of using the learning tool. All items were measured using a five-point Likert-type scale with anchors from "Strongly disagree $=1$ " to "Strongly agree $=5$ ".

Over a period of five years teaching the MIS course in a face-to-face mode, and then creating the onlince version of it, we consistently found out that many students desired and were open to use any method provided to them in order to help them learn the course content. They were motivated to do extra work to enhance their chances for a better grade. This is especially true probably due the nature/context of our university in Montreal, Canada where our student population was very international and diverse. At the same time, the university was very systematic in investing into the computer-assisted instructions. The context of our educational institution entailed a diverse group of students:

- from English, French, Asian and Middle Eastern backgrounds (this alone presenting major implications in relation to English proficiency which is the language of the instruction at our University),

- with an educational background ranging from high school to college graduates,

- where the majority work part-time and even full-time, thereby trying to manage both in their lives,

- where a significant number of them have to commute at least 45 minutes to campus, and,

- where a large number have not decided on their major.

In response to the student desire for learning support and considering the challenges at that time, the learning tool was piloted. After a three cycles of refinements over three semesters, all student, IT, and learning issues were resolved and the final system and process described above was frozen for use and evaluation.

All our experiences in relation to students using this learning tool pointed towards the persistence of anxiety which also varied in type and in level across different student groups (at least that was our initial general observation by interacting with the students). Motivated to get insight into the students' experiences of anxiety we decided to study it as it relates to computer and internet experience. Moreover, our aim of the study is to understand how these anxieties differ across various student group. To that effect, this study focuses on comparing the middle easter group with the western group.

It is important to note that the leaning tool was used in a mandatory setting where the student practice scores counts towards the course final grade, hence introducing another type of anxiety that is even when students are practicing. We did this because we wanted to encourage the students not to only complete 20 questions for practice but do much more. To mitigate this anxiety, the running score of the practice would count.

We acknowledge the complex nature of anxiety and that this complexity cannot be captured fully in this study. However, in this attempt we decided to study overall computer anxiety with one item (ANX3) to be more specific to the present context where students can report on their fear of making mistakes that cannot be corrected and hence providing a direct link to performance in the course. 


\section{Discussion \& Analysis of Results}

The usable questionnaires were examined for missing data and a mean substitution was used to generate replacement values. Missing data is frequently encountered in empirical research and can be treated in a number of ways such as the case where the missing data of non-sampled observations can be ignored from the analysis. In general, the justification for allowing missing data to be ignored is that it is operating at random or is explicitly addressed by the techniques used. Deleting an entire respondent's record is acceptable whenever missing data is completely at random. To that effect, in our analysis we found that the missing data does not seem to be completely random and have decided to use simplest means in replacing the missing values (Hair et al., 1998).

Table 2 presents the mean and standard deviations for CK, IUse, TDIUse, and ANX items. Also, the last column presents the difference in means between the Arabic and English groups, in percent. The results in the table show that both groups have the same perceptions about their computer knowledge and time of using the internet every day. However it it seems that Arabic students tend to use the Internet daily 11 percent more than English speaking students.

Table 2: Descriptive statistics.

\begin{tabular}{|l|c|c|c|c|c|}
\cline { 2 - 5 } \multicolumn{1}{c|}{} & \multicolumn{2}{|c|}{ Arabic (N=54) } & \multicolumn{2}{c|}{ English (N=217) } & \multicolumn{1}{c}{} \\
\cline { 2 - 5 } \multicolumn{1}{c|}{} & Mean & $S D$ & Mean & $S D$ & $4, \%$ \\
\hline CK & 3.52 & 0.95 & 3.61 & 0.90 & 2 \\
\hline IUse & 2.67 & 0.75 & 3.00 & 0.68 & 11 \\
\hline TDIUse & 3.15 & 1.04 & 3.16 & 0.97 & 0 \\
\hline & & & & & \\
\hline ANX1 & 3.0 & 0.9 & 2.3 & 1.2 & -30 \\
\hline ANX2 & 2.6 & 1.2 & 2.0 & 1.2 & -30 \\
\hline ANX3 & 1.8 & 1.0 & 1.6 & 0.9 & -13 \\
\hline ANX4 & 1.1 & 1.1 & 1.7 & 1.0 & 35 \\
\hline
\end{tabular}

More importantly, table 2 shows large differences between the two groups in relation to the anxiety items. It is clear from the table that the Arabic group feel more apprehensive about using computers (ANX1), are more scared to cause computer problems by hitting the wrong key (ANX2) and are more hesitant to use a computer for fear of making mistakes that they cannot correct (ANX3) than the English group, by 30\%, 30\% and 13\% respectively. However, they are seem to be less intimidated by computers than the English group by $35 \%$. This is somewhat interesting that Arabic students are more worried about using computers but less intimidated by them than the English group. This may be due to motivational mechanisms and requires further investigation.

Figures 1 to 4 present a comparison of the four anxiety items between the Arabic and English groups. In these figures, the y-axis shows the likert scale where 1 is strongly disagree and 5 is strongly agree. The x-axis shows the frequency of answers on each answer on the likert scale. The sum of each category (Arabic, and English) should total 100\%.

Looking at the four figures below we can observe general differences on the scale $1=$ 'strongly disagree' and $3=$ 'neutral'. and considering only the scores, we can observe the following: (1) 
There are four times as many Arabic students as English who reported to be more apprehensive about using computers; This difference seems to be shifted to scale $3=$ neutral. (2) Similar results are found in item ANX2 (figure 2) which gives a subjective measure of students being scared to make keyboard typing mistakes. This may be due to language issues and lack of computer experience influencing their confidence. (3) Regarding students being hesitant to use a computer for fear of making mistakes that cannot be fixed, ANX3 (figure 3), there is no important difference between the two groups; and finally (4) The opposite is observed from figure 4, where the English group seems to be more intimidated with computer than the Arabic group.

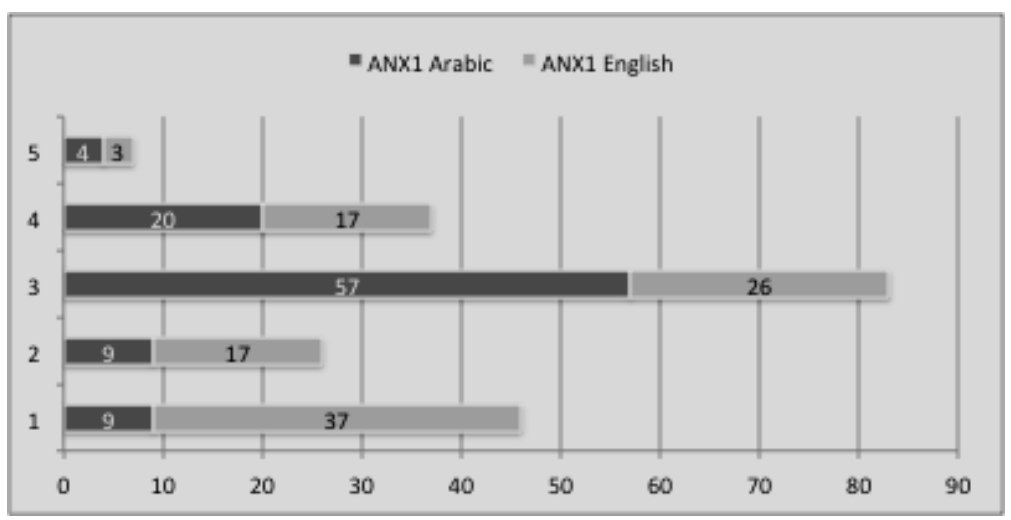

Figure 1: ANX1 comparisons.

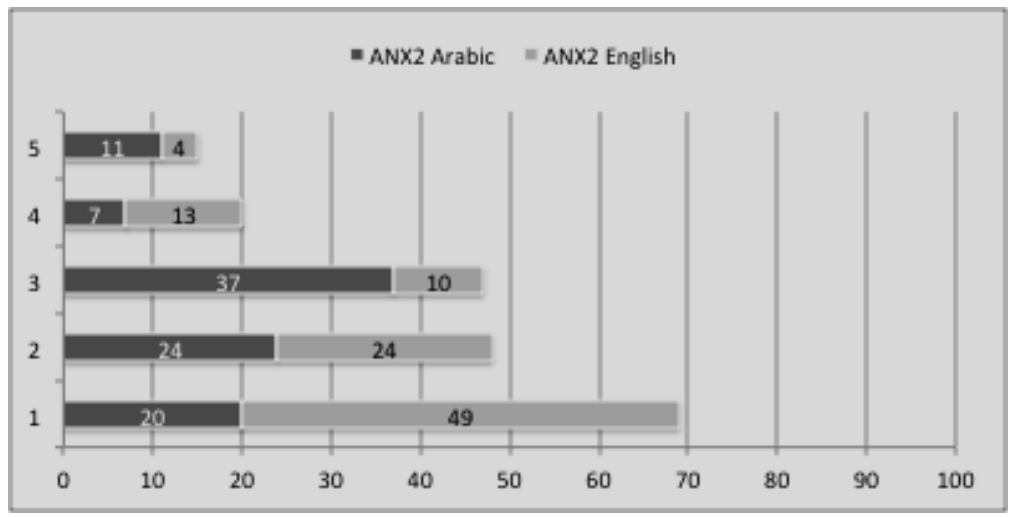

Figure 2: ANX2 comparisons.

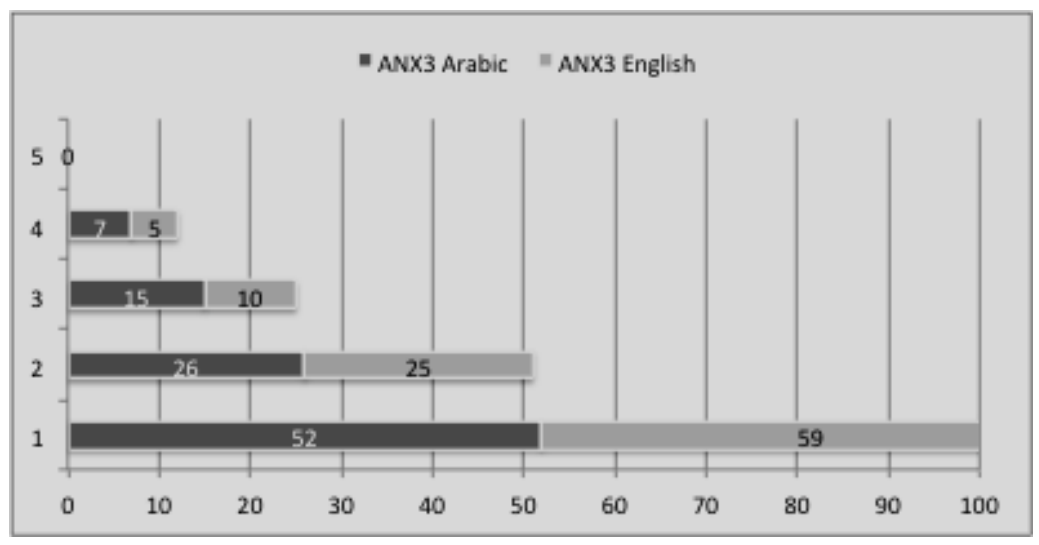

Figure 3: ANX3 comparisons. 


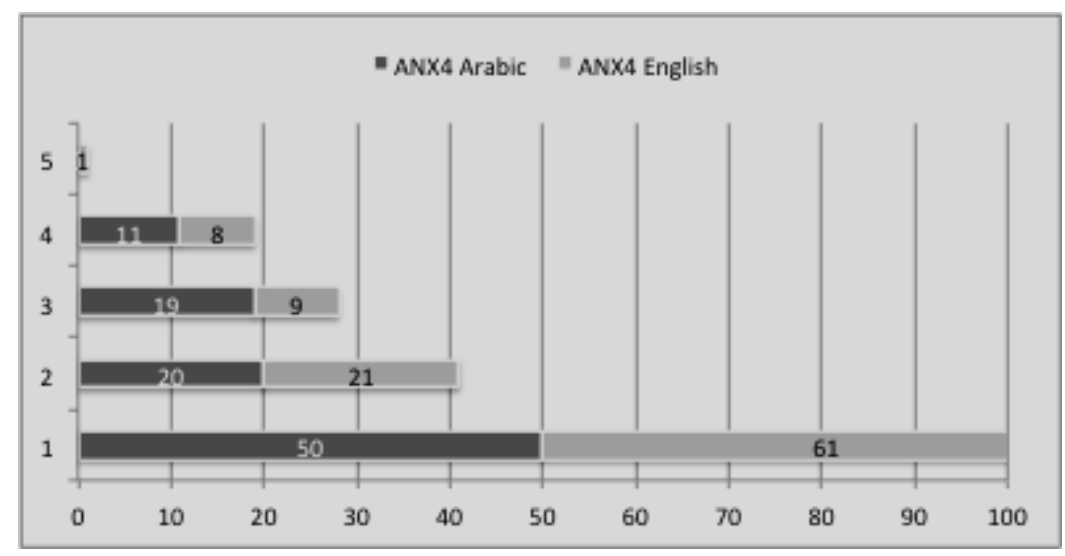

Figure 4: ANX4 comparisons.

It is evident that there are differences in the notions of computer anxieties as reported by the students, namely in the emotions of apprehension, scaredness, hesitation and intimidation. The differences between the two groups among the anxiety emotional scales are summarized as follows:

- Apprehension:

- Scared to make errors:

- Hesitatation \& fear to make mistake

- Intimidation

$$
\begin{aligned}
& \text { Arabic }>\text { English } \\
& \text { Arabic }>\text { English } \\
& \text { Arabic }=\text { English } \\
& \text { Arabic }<\text { English }
\end{aligned}
$$

Next, we looked into the Cronbach's Alpha. The Cronbach's alpha coefficient is analysed for internal consistency reliability of the items measuring the anxiety construct. As summarized in Table 3, the reliability of most of all constructs were acceptable (Nunnally, 1978). The Cronbach's Alpha for the Arabic group is lower than desired, however this may be due to the sample size.

Table 3: Cronbach's Alpha (CA) analysis.

\begin{tabular}{|l|c|c|}
\hline & $\begin{array}{c}\text { Arabic (N=54) } \\
\text { Cronbach's Alpha (0.58) }\end{array}$ & $\begin{array}{c}\text { English (N=217) } \\
\text { Cronbach's Alpha (0.79) }\end{array}$ \\
\cline { 2 - 3 } & $\begin{array}{c}\text { Omitted Item Stats } \\
\text { Omitted Item Stats }\end{array}$ \\
\hline ANX1 & 0.63 & 0.82 \\
\hline ANX2 & 0.57 & 0.75 \\
\hline ANX3 & 0.36 & 0.71 \\
\hline ANX4 & 0.45 & 0.67 \\
\hline
\end{tabular}

At the same time, we performed additional analysis on the Cronbach's Alpha by recalculating the reliabilities of the items on the anxiety construct while removing one item at a time (Saadé and Bahli, 2005). For example in the first row of table 3, with the label ANX1, this row tells us that if ANX1 is removed the Cronbach's Alpha for the Arabic group would be 0.63, and for the English group 0.82. The table shows that for best Cronbach's Alpha results, the removal of the ANX1 is desired giving the highest values. 
The performance of CA is largely attributable to the reliabilities of the items that comprise a scale, and also to the amount of systematic error and to the sample size. Generally, there is a strong positive association between the level of systematic error and size. Considering that the variability of the sampling distribution of correlations is negatively related to sample size, the calculation of the CA then, which is based on correlations, is expected to decrease as sample size increases. This provides the basis for further analysis of the Arabic group relatively low CA value.

Table 4: Regression analysis.

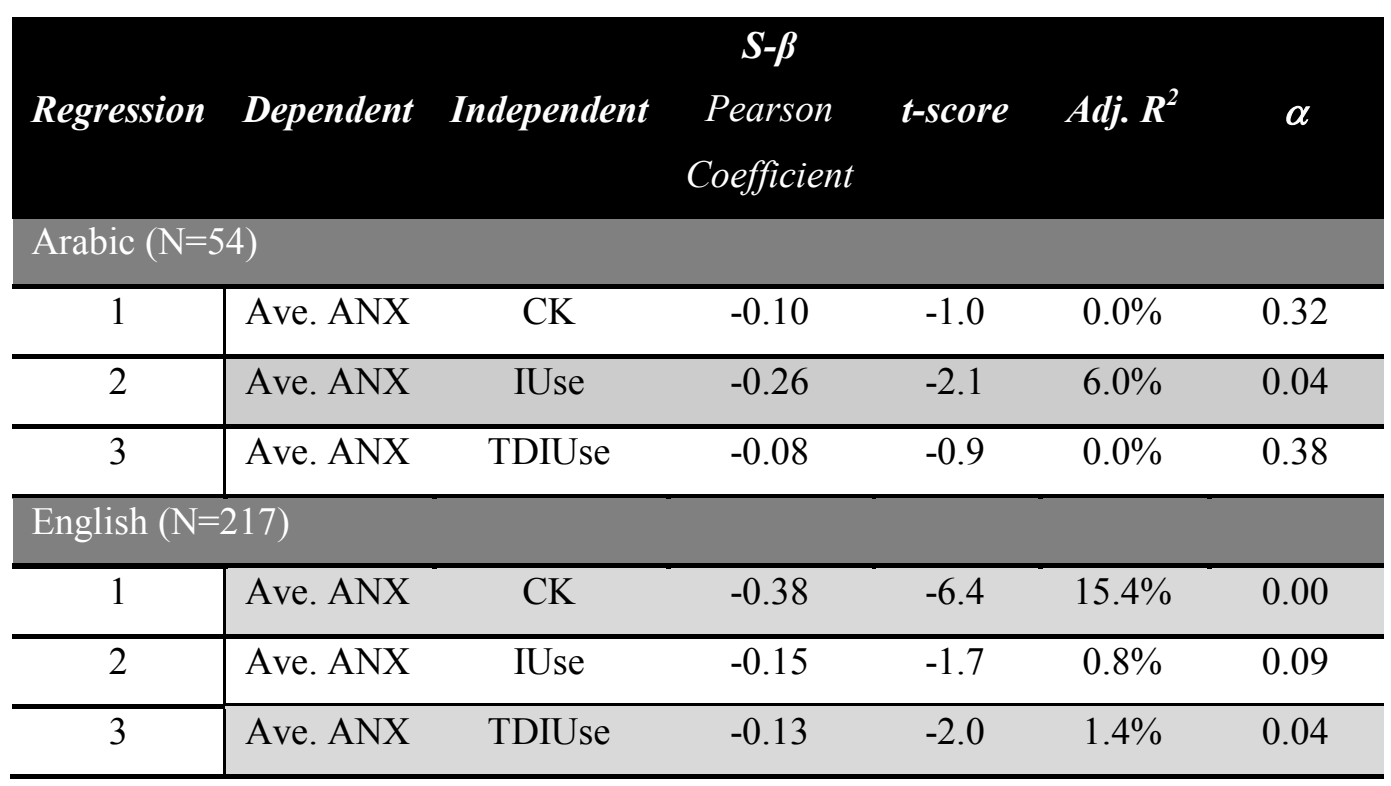

S- $\boldsymbol{\beta}$ : Standardized Beta Coefficient; $\boldsymbol{\alpha}$ : Significance.

As a final analysis, we ran some regression analysis to understand the relationship between computer experiences, namely CK, IUse and TDIUse as shown in table 4 above. The highlighted rows reveal the relationships that were found to be significant with an alpha value less than 0.05 . These relationships indicate that internet usage predicts anxiety in the Arabic group of students while computer knowledge and time duration of internet use impacts the English group. All significant relationships for both groups have a negative impact pearson coefficient indicating that an increase in the independent variables (computer and internet amount of experience, in general) influences anxiety by reducing it.

\section{Conclusions}

Analysis of the results demonstrate that there are important differences in anxiety between the Arabic and the English groups of students. At the same time, our analysis shows that computer and internet experience impact the anxieties of these two groups differently. Specifically, our analysis indicates that the anxiety construct as measured by the emotions of apprehension, being scared, hesitation and intimidation (of course as they relate to computer usage in the leaning context) is best evaluated without the item related to the 'feeling of apprehension of using computers'.

Moreover, knowledge of computers, frequency of internt usage, and time duration of using the internet influence anxieties of the two groups differently. Frequency of internet usage (IUse) explains to a small extent $(6 \%)$ the anxieties in the Arabic group, while self reported computer 
knowledge (CK) explains around 15\% of the anxieties found in the English group of students. Time duration using the internet (TDIUse), although has a significant influence on anxieties for the English group, the $\mathrm{R}^{2}$ values is $1.4 \%$ thereby showing negligible explanation power. So in conclusion, we can identify Internet frequency of usage and subjective evaluation of computer knowledge as the two distinctive variable to influence the Arabic and English groups respectively.

\section{Limitations \& Future Research}

We wish to acknowledge the limitations in this Study. This study was done in the context of online learning for an introductory course in a school of business. This which may be viewed as a limitation calls to be careful to generalize the results to other types of academic institutions and other countries where the national culture may have different effects on students from various cultures. At the same time, the sample size was for the Arabic group is limited. Sample size should be increased to ensure more reliable results. Also, the anxiety and computer/Internet experience items should be expanded in scale to cover other academic institutions in different countries with a significantly larger number of and more diverse participants to ensure the reliability of our findings.

In terms of future research, we identify a number of opportunities that are necessary to strengthen the body of knowledge. The body of literature today is weak in terms of cultural studies in online learning environments, as it lacks not only learning frameworks and models that explain student's experience and success, but also the breadth and depth of learning theory using information technologies for learning purposes. In essence, online learning using web-based tools and associated management technologes have not been properly addressed and the body of knowledge remains superficial and online learning management is hardly ever simple and rather always complex.

We would like to highlight that the notion of learning success using online learning environments is not well understood and many definitions have been proposed, yet they are still close to those used for the traditional classroom. Most research work make an attempt to define student learning success where they anchor their results to the traditional notions of the student-teacher classroom. Maybe more appropriate definitions and adequate quality needs to be identified. Is learning success as we define it today really important? What is a successful student? There are many cases (and which the first author had observed and even taken part in) where the academic failure of a student was actually a significant portion of the formula for his/her career and life success. We stress the need to depart from the traditional student-teacher classroom notion of learning success and find other learning qualities that measure more appropriate outcomes.

\section{References}

Aldalalah, O. A., \& Gasaymeh, A. M. M. (2014). Perceptions of blended learning competencies and obstacles among educational technology students in light of different anxiety levels and locus of control. Contemporary Educational Technology, 5(3).

Al-Gahtani, S. S. (2014). Empirical investigation of e-learning acceptance and assimilation: A structural equation model. Applied Computing and Informatics.

Ayersman, D. J., \& Reed, W. M. (1995). Effects of learning styles, programming, and gender on computer anxiety. Journal of Research on Computing in Education, 28(2), 148-161.

Brosnan, M. J. (1998). Technophobia: The psychological impact of information technology. New York: Routledge.

Davis, F. D. (1989). Perceived usefulness, perceived ease of use, and user acceptance of information technology. MIS Quarterly, 13(3), 319-339. 
Igbaria, M., \& Chakrabarti, A. (1990). Computer anxiety and attitudes towards microcomputer use. Behaviour and Information Technology, 9(3), 229-241.

McInerney, V., McInerney, D. M., \& Sinclair, K. E. (1994). Student teachers, computer anxiety, and computer experience. Journal of Educational Computing Research, 11(1), 27-50.

Nunnally, J. C. (1978). Psychometric theory (2nd ed.). New York: McGraw-Hill.

Pedersen, P.. \& Nysveen, H. (2003): Usefulness and self-expressiveness: Extending Reed, W. M., Ayersman, D. J., \& Liu, M. (1996). The effect of students' computer-based prior experiences and instructional exposures on the application of hypermedia-related mental models. Journal of Educational Computing Research, 14(2), 175-197.

Rosen, L. D., Sears, D. C., \& Weil, M. M. (1987). Computerphobia. Behavior Research Methods, Instruments, \& Computers, 19(2), 167-179.

Saadé, R. G., \& Bahli, B. (2005). The impact of cognitive absorption on perceived usefulness and perceived ease of use in on-line learning: An extension of the technology acceptance model. Information \& Management, 42(2), 317-327.

Saadé, R. G., \& Kira, D. (2007). Mediating the impact of technology usage on perceived ease of use by anxiety. Computers \& Education, 49(4), 1189-1204.

Venkatesh, V. (1999). Creation of a favorable user perceptions: Exploring the role of intrinsic motivation. MIS Quarterly, 23(2), 239-260.

\section{Biographies}

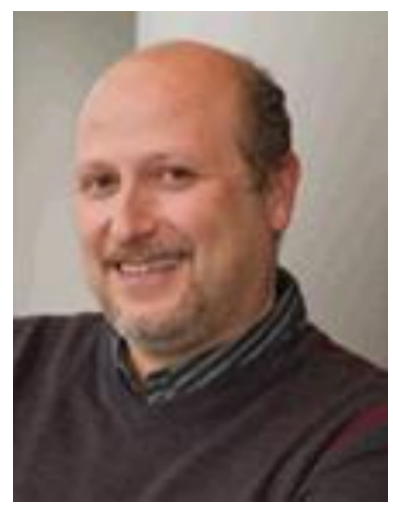

Dr. Raafat George Saadé has been teaching in the faculty since 1998. He obtained his Ph.D. in 1995 (Concordia University) after which he received the Canadian National Research Council postdoctoral fellowship, which he completed at McGill University in Montreal. Dr. Saadé has been engaged in consultancy since 2000 locally and internationally. Dr. Saadé has published in journals such as Information \& Management, Decision Sciences, Decision Support Systems, Computers \& Education, and Expert Systems with Applications. His research interests include the development and assessment of information systems, and the supply chain of digital information products.

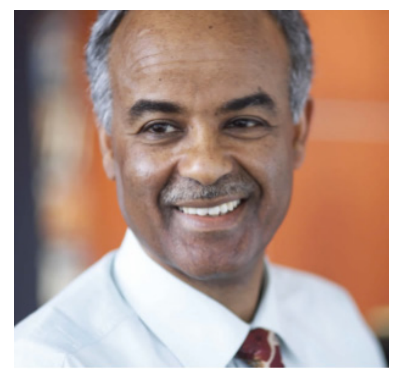

Fassil Nebebe (Ph.D., P.Stat.) is a Professor and Chair of the Department of Decision Sciences \& MIS of the John Molson School of Business, Concordia University, Montreal, Canada. His research focuses on statistical methods using Bayesian modeling and has made contributions with results published in different areas such as small area estimation, the bootstrap, Gibbs sampling, statistical computing, regression models and simulated MLE. He has served as Managing Editor of Liaison - The Newsletter of the Statistical Society of Canada (2004 2007), and as President of the Statistical Society of Montreal

(2002/2003). He is the founding President of SSENA (1999-2002). 


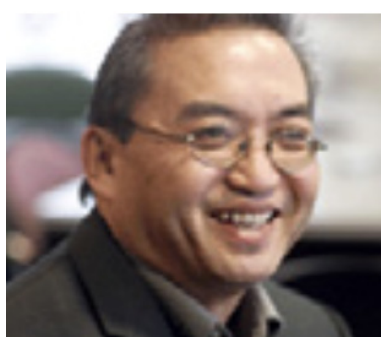

Dr. Dennis Kira is an associate professor at the Decision Sciences MIS department, John Molson School of Business, Concordia University, Canada. He has been with the Faculty since 1983. He obtained his Ph.D. from University of British Columbia. Dr. Kira teaches System design, decision support systems, data management, data mining, Internet related programming, and e-commerce His research activities include Ecommerce, web design, distance learning, decision making under uncertainty, neural networks, machine learning, and financial modeling. Dr. Kira has published in IEEE, ORQ and management science among other journals. 